\title{
Wellens' Syndrome: An Atypical Localization After an Atypical Transient Left Bundle Branch Block Presentation
}

\author{
John C. Yang ${ }^{\mathrm{a}, \mathrm{d}}$, Erica Kuhn ${ }^{\mathrm{a}}$, Parvataneni Arun ${ }^{\mathrm{b}}$, Serap Sobnosky
}

\begin{abstract}
Wellens' syndrome is a clinical presentation with a particular pattern of electrocardiographic (ECG) T-wave changes associated with critical, proximal left anterior descending (LAD) coronary artery lesions. We report a case of a variant presentation of Wellens' syndrome diagnosed after the resolution of a transient left bundle branch block (LBBB). The patient initially presented with acute coronary syndrome (ACS), which failed to be diagnosed despite LBBB presentation, but later became apparent after ECG converted to a Wellens' type pattern. There are three major learning points from this case. First, Wellens' syndrome can be difficult to recognize when presenting with an abnormal initial ECG-in our case it was only diagnosed after LBBB resolution. Second, Wellens' syndrome can present with atypical localization of the offending lesion-our patient was ultimately identified to have a mid-LAD lesion. Third, there is no data in the literature regarding the time to T-wave recovery after revascularization of the LAD-our follow-up marks the timing at somewhere between 2 weeks and 4 months.
\end{abstract}

Keywords: Wellens' syndrome; Left bundle branch block; Left anterior descending; T-wave normalization

\section{Introduction}

Wellens' syndrome is a clinical presentation with a particular pattern of electrocardiographic (ECG) T-wave changes associated with critical, proximal left anterior descending (LAD)

Manuscript submitted June 6, 2019, accepted June 24, 2019

${ }^{a}$ College Medical Center, Graduate Medical Education Office, 2776 Pacific Ave, Long Beach, CA 90806, USA

${ }^{\mathrm{b}}$ Cardiology Department, Long Beach Memorial Medical Center, 1050 Linden Ave, Long Beach, CA 90813, USA

${ }^{\mathrm{C} C a r d i o l o g y}$ Department, St. Mary Medical Center, 2865 Atlantic Ave Ste 104, Long Beach, CA 90806, USA

${ }^{\mathrm{d}}$ Corresponding Author: John C. Yang, College Medical Center, Graduate Medical Education Office, 2776 Pacific Ave, Long Beach, CA 90806, USA.

Email: drjohncyang@gmail.com

doi: https://doi.org/10.14740/jmc3322 coronary artery lesions. Without emergent revascularization, many will go on to develop extensive anterior wall infarction, even with medical management. We report a case of a variant presentation of Wellens' syndrome diagnosed after the resolution of a transient left bundle branch block (LBBB), with a variant finding of a mid-LAD lesion on angiography, and with a previously not described in medical literature timing for Twave normalization of somewhere between 2 weeks and 4 months.

\section{Case Report}

A 68-year-old man presented to the emergency room with sudden onset of $9 / 10$ retrosternal chest pain with radiation to his back associated with severe shortness of breath that began $3 \mathrm{~h}$ prior to arrival. Patient denies similar past episodes but does admit influenza-like symptoms for the preceding 3 days. Patient's medical history includes hypertension and diabetes mellitus type 2, he takes no medication and has not received medical follow-up for more than 3 years. Social history is significant for smoking history of $40+$ pack-years.

On presentation, patient was in severe respiratory distress requiring bilevel positive airway pressure (BiPAP) support. Patient had an initial heart rate of 140 , respiratory rate of 34 , blood pressure of $182 / 92$, with oxygen saturation of $89 \%$ on $40 \% \mathrm{FiO} 2 \mathrm{BiPAP}$. Chest X-ray was significant for pulmonary edema and ECG revealed a LBBB-there were no prior ECGs available for comparison (Fig. 1). Initial labs were significant for troponin of 0.11 , BNP of 761, WBC of 14.5 and lactic acid of 4.0. Echocardiography revealed grade I left ventricular diastolic dysfunction with apical akinesia and septal dyskinesia, and an ejection fraction (EF) of approximately $30 \%$. Re-examination at $1 \mathrm{~h}$ reveals an ECG that remains relatively unchanged whereas patient's chest pain and shortness of breath have resolved (Fig. 2). Patient was considered for emergent cardiac catheterization; however patient was refused by STelevation myocardial infarction (STEMI) receiving center. Ultimately, the patient was admitted to the intensive care unit (ICU) for close monitoring and rule out acute coronary syndrome (ACS) versus pulmonary edema with cardiomyopathy.

Continuing care in the ICU, serial troponins peaked at 0.49 with subsequent downtrending, and serial ECGs showed persistent LBBB. Free of chest pain and with symptoms of short- 


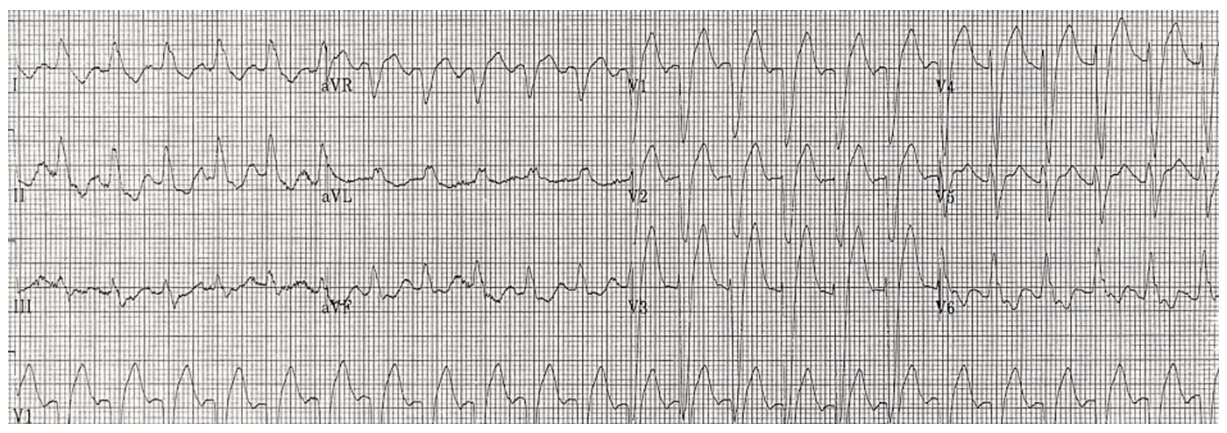

Figure 1. ECG initial presentation.

ness of breath resolved, the plan was to discharge the patient with urgent outpatient cardiology follow-up. However, ECG at $33 \mathrm{~h}$ revealed resolution of $\mathrm{LBBB}$ with new deeply inverted Twaves in leads V2-3-4 (Fig. 3); troponins at this time remained unalarming at 0.37 . The Wellens' pattern T-waves indicated a severe proximal LAD lesion, thus prompting an emergent transfer to a percutaneous coronary intervention (PCI) capable hospital.

The patient underwent coronary angiography and was found to have single vessel coronary artery disease, reported as $99 \%$ focal stenosis in the mid-portion of the LAD (Fig. 4). The patient underwent a successful transluminal coronary angioplasty with stent deployment. Follow-up ECG at 2 weeks showed unchanged deep symmetrical inverted T-waves (Fig. 5), whereas repeat ECG at 4 months revealed normalized findings (Fig. 6). Follow-up echocardiography at 6 months revealed normal wall motion with an EF of 55-60\%. Written consent for publication was obtained from patient and on record at cardiologist's office. Ethics approval is not required at this institution for case reports.

\section{Discussion}

In 1982, Wellens published his observation that a pattern of inverted T-waves in the precordial leads was strongly associ-



Figure 2. ECG $1 \mathrm{~h}$ after. ated with early large anterior myocardial infarction (MI) and a poor prognosis in patients presenting with unstable angina. He further demonstrated that most of these patients had significant disease of the proximal LAD, and that without coronary revascularization $75 \%$ went on to develop extensive anterior wall infarction within weeks, even with medical management [1]. Consequently, proper diagnosis and early cardiac catheterization with subsequent angioplasty or coronary bypass surgery are indicated for these patients [2]. Proper recognition of Wellens' syndrome is critical as exercise stress tests in these patients can lead to infarction at the time of increased cardiac demand and should be avoided [3].

The key diagnostic features of Wellens' syndrome are the T-wave findings which can present in one of two patterns. Wellens' type A (also known as type II) has a biphasic pattern, as seen in lead V2 in Figure 3, and represents 25\% of cases. Wellens' type B (also known as type I) is deeply inverted with symmetric contours, as seen in lead V3-4 in Figure 3, and is the more common variant representing $75 \%$ of cases seen. These ECG changes are usually seen in the mid precordial leads and classically manifest during the pain-free state immediately after angina resolution. Other diagnostic criteria for Wellens' syndrome are as seen in Table $1[3,4]$.

In the described case, the patient had numerous risk factors, a typical presentation of chest pain, elevated troponins with decreased EF, and an unknown chronicity of LBBB-he likely would have benefited from emergent cardiac catheterization. However, the patient was refused by the STEMI receiving center. Given the patient's symptomatic resolution by time of admission along with lack of significant troponin elevation, further attempts to transfer were not pursued. Of note, one

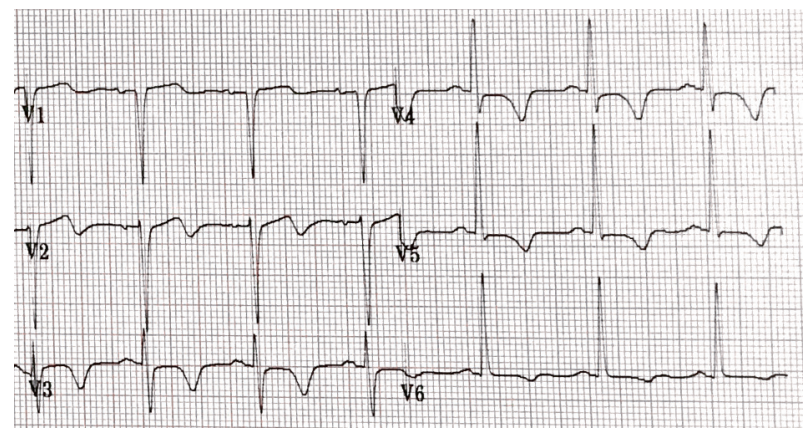

Figure 3. ECG $33 \mathrm{~h}$ after. 

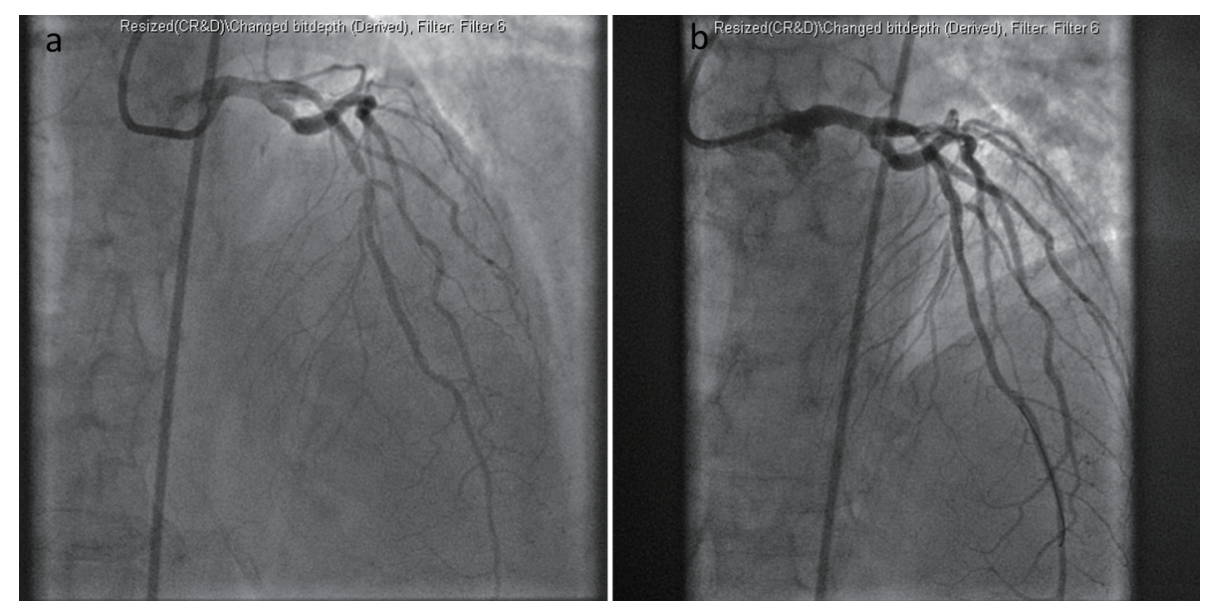

Figure 4. Coronary angiography with stent deployment.

would have expected a much higher troponin level during an event of true ACS resulting in pulmonary edema and an EF of $30 \%$, prompting us to consider this a more chronic congestive heart failure picture. Additionally, ECG performed on initial presentation during the angina phase showed LBBB which did not meet modified Sgarbossa criteria $[5,6]$. ST/S ratios in V2 and V3 of the initial ECG (Fig. 1) are approximately 2/13 and $5 / 27(-0.15$ and -0.19$)$, and no worse in the 1-h repeat ECG (Fig. 2). Furthermore, were this the typical Wellens presentation, the T-wave inversion should have become apparent during the painless phase on the 1-h repeat and 9-h repeat ECGs, with or without LBBB obscuration [7]. All that being said, had this patient presented to a PCI capable hospital initially, he would likely have undergone coronary angiography. However, being a smaller community hospital without PCI capabilities and without a willing STEMI receiving center, transferring asymptomatic patients simply for LBBB of unknown chronicity becomes exceedingly difficult.

Trending serial troponins and serial ECG, the ECG at 33 h (Fig. 3) no longer showed LBBB pattern, but instead

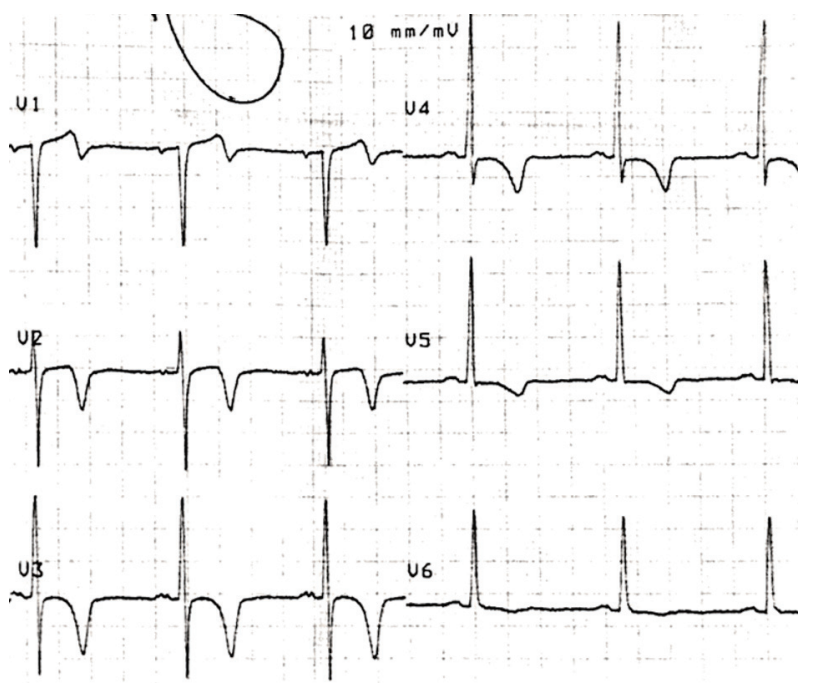

Figure 5. ECG 2 weeks after. showed T-wave changes consistent with Wellens' syndrome. Although the differential of T-wave inversion after LBBB does include memory T-waves, this was not thought to be the case due to the patient's clinical presentation [8]. In hindsight, the transient LBBB likely represents a transient subepicardial ischemic event, which then spontaneously opened up resulting in the patient's symptomatic improvement, finally with time allowed for the Wellens' type T-wave inversions to develop. In our literature search, there have been no reports of confirmed Wellens' syndrome diagnoses made in association with the resolution of a transient LBBB. This atypical presentation of Wellens' syndrome is important to report as it can represent delayed manifestations and thus reinforces the value of monitoring serial ECGs in addition to serial troponins, which in this case had been steadily downtrending since admission.

Following his hospital transfer, the patient underwent cardiac catheterization and the diagnosis for Wellens' syndrome was confirmed with a focal lesion in the mid-portion of the LAD. There have been other reports of atypical presentations of Wellens' syndrome which also report the focal lesion as be-

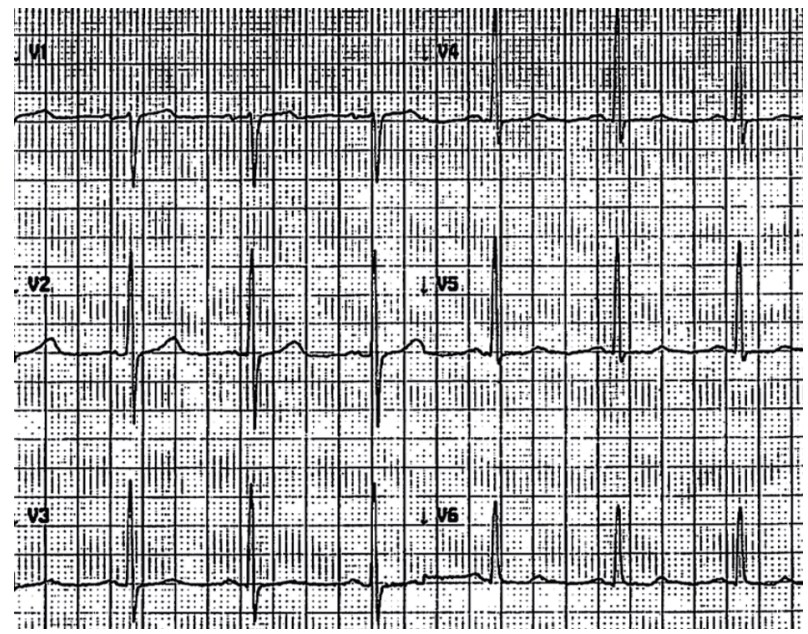

Figure 6. ECG 4 months after. 
Table 1. Criteria for Wellens' Syndrome

\section{Criteria for Wellens' syndrome}

Wellens' type A or type B wave(s) in leads V2 and V3, occasionally in leads V1-V6

Recent history of angina

Pattern present during pain-free state

Isoelectric or minimally elevated $(<1 \mathrm{~mm}) \mathrm{ST}$ segment

No precordial Q waves and preserved $\mathrm{R}$ wave progression

Normal or slightly elevated cardiac serum markers

ing in the mid-portion of the LAD as opposed to the proximal [9].

Outpatient cardiology follow-up for this patient revealed normalization of T-waves somewhere between 2 weeks and 4 months. Our literature search revealed a lack of data regarding the timing of T-wave normalization after successful coronary interventions for Wellens' syndrome. As persistent T-wave inversion can present as a potential source of confusion and medical error much like in memory T-waves, we hope to see further study in regards to the timing of ECG normalization in patients with Wellens' syndrome.

\section{Conclusions}

The critical nature of recognizing the ECG pattern of Wellens' syndrome is well established in the medical community. Failure to recognize Wellens' syndrome, which is a pre-infarction stage of coronary artery disease, can lead to deleterious outcomes-whether by inadequate treatment or by inappropriate stress testing. Our case report describes an atypical presentation of Wellens' syndrome which revealed itself on serial ECG long after chest pain resolution and only after resolution of the transient episode of LBBB found on initial ECG. This delayed manifestation is noteworthy and justifies continued vigilance on the part of medical practitioners in reviewing serial ECGs in cardiac patients. Additional observations were made regarding the mid-LAD locale of the offending lesion, and regarding the length of time for T-wave normalization marked somewhere between 2 weeks and 4 months.

\section{Acknowledgments}

None.

\section{Financial Disclosure}

This research did not receive any specific grant from funding agencies in the public, commercial, or not-for-profit sectors.

\section{Conflict of Interest}

None.

\section{Informed Consent}

Written consent for publication was obtained from patient and on record at cardiologist's office.

\section{Author Contributions}

JY contributed to the care of the patient, acquisition of data, analysis and interpretation of data, drafting and revising the article, and communication as corresponding author; EK contributed to supervision of above physician in research endeavors as an Internal Medicine Program Director, and minor editing of the manuscript; PA contributed to the care of the patient, and minor editing of the manuscript; SS contributed to the care of the patient, acquisition of data, analysis and interpretation of data, and assistance with editing and revising the manuscript.

\section{References}

1. de Zwaan C, Bar FW, Wellens HJ. Characteristic electrocardiographic pattern indicating a critical stenosis high in left anterior descending coronary artery in patients admitted because of impending myocardial infarction. Am Heart J. 1982;103(4 Pt 2):730-736.

2. de Zwaan C, Bar FW, Janssen JH, Cheriex EC, Dassen WR, Brugada P, Penn OC, et al. Angiographic and clinical characteristics of patients with unstable angina showing an ECG pattern indicating critical narrowing of the proximal LAD coronary artery. Am Heart J. 1989;117(3):657665.

3. Rhinehardt J, Brady WJ, Perron AD, Mattu A. Electrocardiographic manifestations of Wellens' syndrome. Am J Emerg Med. 2002;20(7):638-643.

4. Tandy TK, Bottomy DP, Lewis JG. Wellens' syndrome. Ann Emerg Med. 1999;33(3):347-351.

5. Smith SW, Dodd KW, Henry TD, Dvorak DM, Pearce LA. Diagnosis of ST-elevation myocardial infarction in the presence of left bundle branch block with the ST-elevation to $\mathrm{S}$-wave ratio in a modified Sgarbossa rule. Ann Emerg Med. 2012;60(6):766-776.

6. Sgarbossa EB, Pinski SL, Barbagelata A, Underwood DA, Gates KB, Topol EJ, Califf RM, et al. Electrocardiographic diagnosis of evolving acute myocardial infarction in the presence of left bundle-branch block. GUSTO-1 
(Global Utilization of Streptokinase and Tissue Plasminogen Activator for Occluded Coronary Arteries) Investigators. N Engl J Med. 1996;334(8):481-487.

7. Grautoff S. Wellens' syndrome can indicate high-grade LAD stenosis in case of left bundle branch block. Herzschrittmacherther Elektrophysiol. 2017;28(1):57-59.
8. Vakil K, Gandhi S, Abidi KS, et al. Deep T-wave inversions: cardiac ischemia or memory? JCvD 2014;2(2):116118.

9. Kardesoglu E, Celik T, Cebeci BS, Cingozbay BY, Dincturk M, Demiralp E. Wellens' syndrome: a case report. J Int Med Res. 2003;31(6):585-590. 